\title{
Quench Characteristics of a Stabilizer-Free 2G HTS Conductor
}

\author{
Edward A. Young, Chris M. Friend, and Yifeng Yang
}

\begin{abstract}
The prospect of medium/high field superconducting magnets using second generation (2G) HTS tapes is approaching reality with continued enhancement in the performance of these conductors. While the cryogenic stability and quench propagation are fundamental issues for the design and safe operation of superconducting magnets, there is insufficient understanding and experimental data for $2 \mathrm{G}$ HTS conductors, in particular for the high field scenario at low temperature $(<77 \mathrm{~K})$ where the current sharing regime is much larger than in low temperature superconductors. The present work includes a systematic characterization of the relevant thermal-electrical properties used for both qualitative discussion and numerical analysis. Direct measurements of one dimensional adiabatic quench initiation and propagation of a stabilizer-free $2 \mathrm{G}$ conductor have been carried out with spatial-temporal recording of temperature and voltage following the deposition of varying local heat pulses to the conductor at different temperatures between $30 \mathrm{~K}$ and $77 \mathrm{~K}$ carrying different transport currents. The minimum quench energy, and the heat generation in the minimum propagation zone (MPZ) have been obtained as a function of temperature and transport current. The results show quench features unique to HTS such as an increasing MPZ with transport current and higher quench energies at lower temperatures. The experimental results are discussed in the context of current sharing over a large temperature range.
\end{abstract}

Index Terms-Quench propagation, 2G HTS.

\section{INTRODUCTION}

$\mathbf{H}$ IGH TEMPERATURE SUPERCONDUCTOR (HTS) insert coils are the only option available for both all-superconducting magnets above $25 \mathrm{~T}$ and cryogen-free magnets operating above $4 \mathrm{~K}$. While material performance and mechanical design remain serious challenges for HTS magnets, cryogenic stability and quench management have also come into sharp focus following recent progress in the 2nd generation (2G) $\mathrm{YBa}_{2} \mathrm{Cu}_{3} \mathrm{O}_{7-\mathrm{x}}$ (YBCO) tapes and $\mathrm{Bi}_{2} \mathrm{Sr}_{2} \mathrm{CaCu}_{2} \mathrm{O}_{8}$ (2212) wires.

Unlike applications at liquid nitrogen temperatures, where thermal stability is typically a minor issue, HTS high field inserts and cryogen-free magnets are designed to operate at low temperatures $(<30 \mathrm{~K})$ for achieving maximum field and current

Manuscript received August 20, 2008. First published June 05, 2009; current version published July 15,2009 . This work was carried out in collaboration with Oxford Instruments Nanoscience and Vector Fields as a part of IMPDAHMA, a Project supported in part by the TSB Technology Programme of the UK, TP/5/ MAT/6/I/H0647BT.

E. A. Young and Y. Yang are with the Institute of Cryogenics, School of Engineering Sciences, University of Southampton, Southampton SO17 1BJ, UK (e-mail: E.A.Young@ @oton.ac.uk; y.yang@soton.ac.uk).

C. M. Friend is with Oxford Instruments NanoScience, Tubney Woods Abingdon Oxon OX13 5QX, UK (e-mail: chris.friend@oxinst.com).

Color versions of one or more of the figures in this paper are available online at http://ieeexplore.ieee.org.

Digital Object Identifier 10.1109/TASC.2009.2018275 performance. The thermal stability of all superconductors is inevitably lowered at low temperatures because of reduced heat capacity and higher operating current density. However, compared to low temperature superconductors (LTS), the quench behavior of HTS at low temperatures is far more complex for the following reasons [1]-[8]. Firstly, the current-sharing regime, fundamental to the development of the normal zone, is extended from just a few Kelvin above $T_{\mathrm{C}}$ for LTS to potentially $100 \mathrm{~K}$ for HTS at helium temperatures. Within this extended currentsharing regime, the highly non-linear E-J characteristics of HTS cannot be approximated by the classical critical state model. Instead of the linear dependence found in LTS, the HTS heat generation becomes a complex nonlinear function of temperature. Secondly, the relevant thermal-electrical properties including resistivity, specific heat and thermal conductivity also exhibit a strong dependence on temperature in the extended current sharing regime. Such coupled nonlinearities have the potential to fundamentally change the behavior of the underlying heat diffusion process during the quench onset and subsequent propagation.

This paper addresses on the quench behavior of a $2 \mathrm{~mm}$ wide $2 \mathrm{G}$ YBCO tape without a copper stabilization copper layer from SuperPower Inc. This conductor was chosen specifically for its expected low thermal stability and moderate current, which simplifies the implementation of the quench measurement procedure. At the same time this conductor still retains the wide temperature range for current sharing and its influence on the HTS quench characteristics, which is the focus of the present work. Compared with previous studies on HTS quench, e.g. [10]-[12], this work not only covers a larger temperature range but also reveals detailed information on the initiation and the dynamics of minimum propagation zone yet to be fully understood.

\section{EXPERIMENTAL}

\section{A. Quench Measurement}

Quench measurements were carried out in a cryocooled cryostat as described in [5]. The sample was thermally anchored at the two current contacts, which were connected via thermal links to the cryocooler head. The temperature of both contacts were controlled using a heater and can be varied between $20 \mathrm{~K}$ and $80 \mathrm{~K}$. A set of hybrid current leads were used for injecting transport current up to $250 \mathrm{~A}$ from room temperature to the current contacts. The high temperature ends of the HTS section were maintained at $77 \mathrm{~K}$ using a liquid nitrogen buffer, which also provided cooling to a radiation shield for the cryostat. The cryogenic design was sufficient for maintaining a stable set temperature between $20 \mathrm{~K}$ and $80 \mathrm{~K}$ at the current contacts throughout the measurements. 


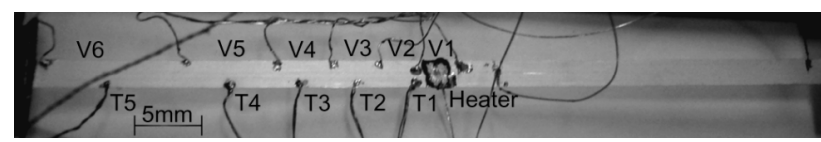

Fig. 1. Picture showing the sample for quench measurements with the heater, thermocouples and voltage taps attached.

The process of quench initiation and propagation was measured using a set of spatially distributed voltage taps and miniature differential thermocouples soldered directly to the sample. The reference junctions of the differential thermocouples were thermally anchored at the current contacts. The electrical and thermal potentials were recorded simultaneously at up to 10 $\mathrm{kHZ}$. The conductor was protected from burning out during quench by limiting the maximum output voltage of the current source and ultimately by a quench protection system to cut off the current source when the overall voltage across the sample exceeded a maximum level (typically $10-100 \mathrm{mV}$ ).

Localized thermal disturbances for quench initiation were applied using short heat pulses (10-100 ms) discharged by a miniature heater made from conductive carbon loaded epoxy. The heater was attached to the conductor using silver-loaded epoxy, and on of the electrodes for the heater was on the sample. The electrical connectivity was used as a direct indication for the thermal connection between the sample and heater. The energy deposited was varied with a resolution up to $0.5 \%$ by changing the level and duration of the electrical pulse. Further details of the experimental procedure can be found in earlier publications [6], [7].

\section{B. Sample}

The HTS sample used in this study is a special $2 \mathrm{G}$ YBCO tape manufactured by SuperPower Inc. Unlike the standard $2 \mathrm{G}$ conductors of $4 \mathrm{~mm}$ width and surrounded by a $20 \mu \mathrm{m}$ thick layer of copper stabilization layer, the present sample is stabilizer free and only $2 \mathrm{~mm}$ wide. The sample has critical currents of $\sim 38 \mathrm{~A}$ at $77 \mathrm{~K}$ and $\sim 400 \mathrm{~A}$ at $4.2 \mathrm{~K}$, within the range where adiabatic quench measurement can be realized without too many complications. The lack of a stabilizer layer significantly reduces the length of the minimum propagation zone, reducing the sample length required.

For the present study, a set of 6 thermocouples and 7 pairs of voltage taps were used, and their layout is shown in Fig. 1. The sample was $6 \mathrm{~cm}$ long, and the heater was located in the center.

\section{RESULTS AND DISCUSSION}

\section{A. Thermal-Electrical Properties}

The quench behavior of HTS at low temperatures is fundamentally influenced by its non-linear thermal-electrical properties over a wide temperature range where the current is shared between the "quenched superconductor" and the resistive matrix. As a precursor to the present study, the specific heat $c_{\mathrm{p}}$, thermal conductivity $k$, and resistivity $\rho$ were measured between $2 \mathrm{~K}$ and $300 \mathrm{~K}$, as shown in Fig. 2. The normal resistivity (solid line) below $T_{\mathrm{c}}$ was obtained using a sample cut from the same length with its superconductivity destroyed by post-processing at $500^{\circ} \mathrm{C}$ in vacuum. The dashed line represents the superconducting transition at $88 \mathrm{~K}$ measured with the as received sample. Below $30 \mathrm{~K}$, the sample shows a residual resistivity of $1.9 \mathrm{n} \Omega \mathrm{m}$,

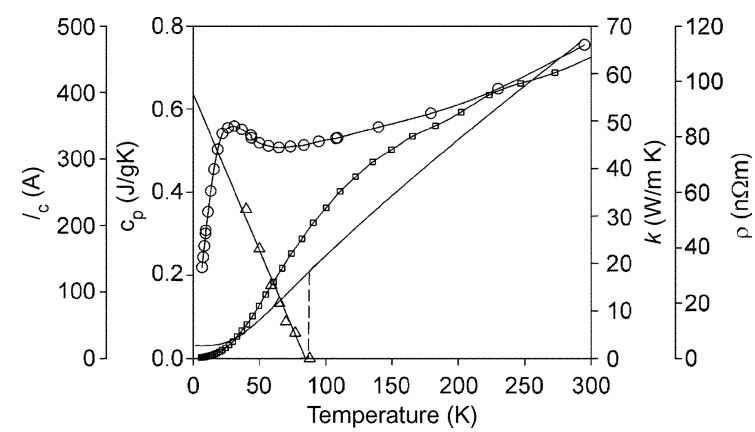

Fig. 2. The critical current $I_{\mathrm{c}}(\triangle)$, specific heat $(\square)$, thermal conductivity (O), and resistivity (solid line) of the $2 \mathrm{G}$ conductor at different temperatures.

i.e. RRR $\sim 60$. The resistivity is mainly attributed to the thin protective Ag layer covering the YBCO film. The specific heat at low temperatures $(<10 \mathrm{~K})$ exhibits a significant linear electronic component attributed to the $\mathrm{Ni}$ in the Hastelloy substrate. The thermal conduction is mainly carried by the silver layer with about $15 \%$ contribution from the substrate [9].

\section{B. Quench Initiation}

For the sample at a given temperature and transport current, the corresponding minimum quench energy (MQE) is determined by measuring the temporal evolution of the hot spot following the application of a heat pulse, which is gradually increased gradually until an irreversible quench is detected. A typical example, with the sample at $40 \mathrm{~K}$ and carrying $212 \mathrm{~A}$ $\left(0.95 I_{\mathrm{C}}\right)$, is shown in Fig. 3, where the temperature $T_{1}$ adjacent to the hot spot and the voltage $V_{1}$ across the hotspot are plotted as a function of time from the start of a 24 ms heat pulse. With a heat pulse of $8.76 \mathrm{~mJ}(\mathrm{~d})$, a peak voltage of $70 \mu \mathrm{V}$ developed across the hot spot after $30 \mathrm{~ms}$, while a peak temperature rise of $\sim 4.8 \mathrm{~K}$ was registered at $T_{1}$ at $100 \mathrm{~ms}$. It should be noted that $T_{1}$ is always lower than the maximum temperature at the middle of the heater, where the temperature is expected to be increased by $30-50 \mathrm{~K}$ after the deposition of about $10 \mathrm{~mJ}$ heater energy (see $c_{\mathrm{p}}(T)$ in Fig. 1). $\Delta T_{1}$ continued to increase after the peak voltage as the energy stored in the hot spot diffused out. The maximum in $T_{1}$ was reached when the voltage across the hot spots had disappeared. Higher heat pulses of $9.25 \mathrm{~mJ}(\mathrm{c})$ and $9.72 \mathrm{~mJ}$ (b) lead to peak voltages of $110 \mu \mathrm{V}$ and $230 \mu \mathrm{V}$, while $T_{1}$ reached maxima of $5.5 \mathrm{~K}$ and $7.1 \mathrm{~K}$ after $120 \mathrm{~ms}$ and $200 \mathrm{~ms}$ by which time $V_{1}$ has almost vanished. An irreversible quench occurred at a heater pulse of $9.97 \mathrm{~mJ}$, with both $V_{1}$ and $T_{1}$ increasing rapidly after $60 \mathrm{~ms}$. It should be noted that a logarithmic time axis is used in Fig. 3 to reveal the details of the voltage/temperature recovery.

The spatial heat diffusion along the sample is illustrated in Fig. 4, which shows the temporal evolution of the voltage and temperature at different locations measured by the spatially distributed voltage taps and thermocouples. For the initial condition of $40 \mathrm{~K}$ and $212 \mathrm{~A}$, it can be seen that $V_{1}, V_{2}$ and $T_{1}$ are in the initial normal zone, and $V_{3}$ is on the outside edge. Within $50 \mathrm{~ms}$ of start of the pulse, the normal zone expands to the size of the corresponding minimum propagation zone (MPZ) to reach just beyond $V_{3}$, i.e., the MPZ length for $40 \mathrm{~K}$ and $212 \mathrm{~A}$ is $l_{\mathrm{MPZ}} \sim 2 \times L_{\mathrm{V} 3}=16 \mathrm{~mm}$. Unfortunately, only one thermocouple $T_{1}$ is within the normal zone. When the normal zone continues to spread beyond the MPZ, an irreversible quench starts 


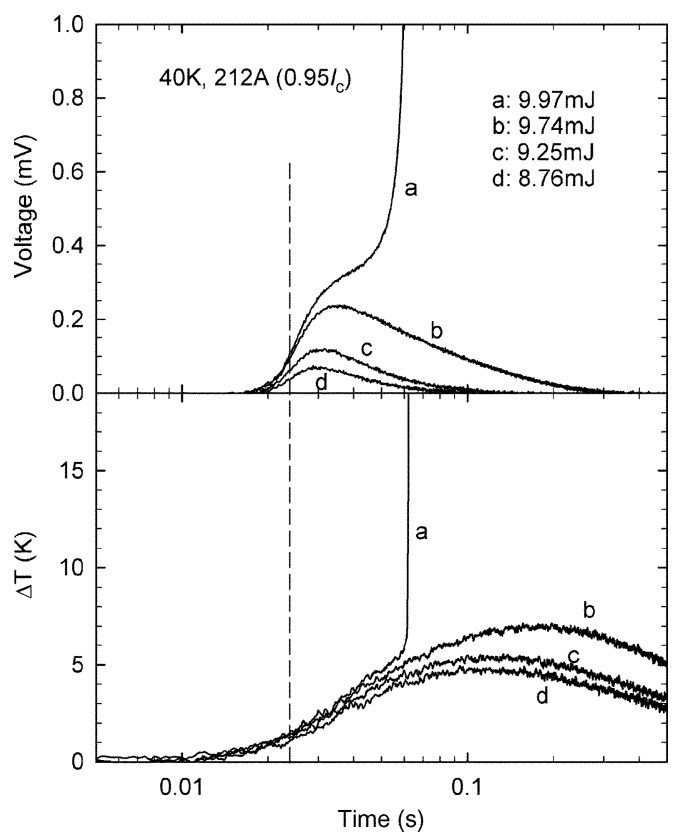

Fig. 3. The hot spot voltage $V_{1}$ and temperature $T_{1}$ as a function of temperature for different heat pulses applied to the sample at $40 \mathrm{~K}$ and carrying $212 \mathrm{~A}$.

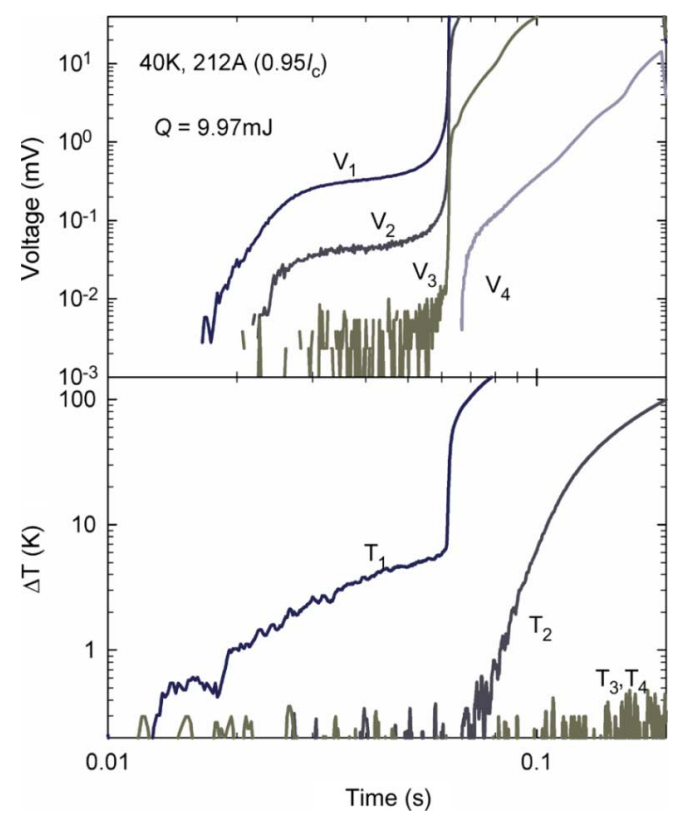

Fig. 4. Normal zone expansion measured by spatially distributed voltage taps and thermocouples. The sample was at $40 \mathrm{~K}$ and carrying $212 \mathrm{~A}$, and the heat pulse is at the MQE of 9,97 $\mathrm{mJ}$.

sharply at $53 \mathrm{~ms}$, with the voltage and temperature rising rapidly from the quasi-MPZ level of $V_{1}=0.4 \mathrm{mV}$ and $\Delta T_{1}=5.2 \mathrm{~K}$.

\section{Current Share and Heat Generation in the MPZ}

According to [7], [8], the size of the minimum propagation zone is determined by the total heat generation within it:

$$
l_{M P Z}=\pi \sqrt{\frac{k(\bar{T})\left(T_{Q}-T_{g}\right)}{G(\bar{T}, J)}},
$$

where $T_{\mathrm{Q}}$ is the maximum temperature in the middle of the MPZ, $T_{\mathrm{g}}$ is the current sharing temperature at which $J_{\mathrm{c}}\left(T_{\mathrm{g}}\right)$ equals the transport current density $J, \bar{T}=1 / 2\left(T_{\mathrm{Q}}+T_{\mathrm{g}}\right)$ is the average temperature of the MPZ, $k$ is the thermal conductivity, and $G(\bar{T}, J)$ is the heat generation in the MPZ in $\mathrm{W} / \mathrm{m}^{3}$.

The quench process in LTS occurs in a narrow temperature range of a few Kelvin of $T_{\mathrm{c}}$, resulting in a maximum MPZ temperature very close to the critical temperature $T_{\mathrm{c}}$, so that the heat generation is well defined by normal resistivity of the matrix, i.e. $G \propto J^{2} \rho_{\mathrm{m}}\left(T_{\mathrm{c}}\right)$. Consequently the well-know MPZ [1] of LTS follows:

$$
l_{M P Z}^{L T S}=\pi \sqrt{\frac{(1-\lambda) k(\bar{T})\left(T_{C}-T_{g}\right)}{\lambda^{2} \rho_{m}\left(T_{c}\right) J^{2}}},
$$

where $J$ is the transport current density in the superconductor and $\lambda$ is the superconductor fill factor in the conductor.

For HTS at intermediate to low temperatures, however, there is a temperature margin of up to $100 \mathrm{~K}$. Within this wide temperature range for quench initiation, the transport current is shared by the superconductor to a varying extent depending on the temperature. As the result, the heat generation in the MPZ is lower than if all the current was carried by normal matrix. Within the wide temperature range $\left[T, T_{\mathrm{c}}\right]$, as shown in [8], the current sharing heat generation $G$ is a highly non-linear function of temperature mainly due to the temperature dependent power-law characteristics of the superconductor, $E \propto\left(J / J_{\mathrm{c}}(T)\right)^{n(T)}$. In principle the MPZ of HTS can be obtained with (1) once the quench temperature $T_{\mathrm{Q}}$ is determined, in practice, an analytic or empirical relation between $T_{\mathrm{Q}}$ and the operating parameters and material properties $\left\{T_{0}, J, E(J), k(T), \rho_{\mathrm{m}}(T)\right\}$ is yet to be obtained. The nonlinearity of $G(T)$ means that even a small error in $T_{\mathrm{Q}}$ will result in a large variation in $l_{\mathrm{MPZ}}$.

The MPZ heat generation can be obtained from the voltage at the center of the hot spot $\left(V_{1}\right)$ when a heat pulse very close to the MQE is applied so that the normal zone expands to quasi-MPZ before quenching irreversibly. An example with the sample at $65 \mathrm{~K}$ is given Fig. 5(a), which shows $V_{1}(t)$ following heat pulses at the MQE for different transport currents from 40 A to 82 A. At small current, e.g. 40 A $\left(48 \%\right.$ of $\left.I_{\mathrm{c}}=83 \mathrm{~A}\right)$, the $V_{1}$ briefly plateaus at $1.9 \mathrm{mV}$ (42 ms from the start of pulse) before quenching rapidly. When the transport current is increased, the quench voltage gradually reduces to reach a minimum of $0.1 \mathrm{mV}$ at $81 \mathrm{~A}(97.5 \% \mathrm{Ic})$, while the time required for the expansion of the initial normal zone to quasi-MPZ grows steadily to $250 \mathrm{~ms}$. Such a large variation in the quench voltage (as indicated by the horizontal lines) and the dynamic of the normal zone initiation are accompanied by a rather modest change $(<1.5 \mathrm{~K})$ in the quench temperature approximated by $T_{1}$ as shown in Fig. 5(b). This correlation of decreasing MPZ heat generation with increasing current has been observed consistently in this sample at different temperatures between $30 \mathrm{~K}$ and $77 \mathrm{~K}$ for up to $97 \%$ of $I_{\mathrm{c}}$. With $T_{\mathrm{Q}}$ only changing moderately with current, a reduction in MPZ heat generation leads to longer MPZ at higher current, as first suggested by numerical solution of the HTS quench diffusion equation [7], [8]. It should be emphasized that the opposite is true for LTS, where the heat generation increases with $J^{2}$. To make the matters even more complex, the heat generation increases again when the current is extremely close to the critical current $\left(>98 \% I_{\mathrm{c}}\right)$.

As expected, the MPZ for this stabilizer-free $2 \mathrm{G}$ conductor is within the measured $6 \mathrm{~cm}$ length of conductor. The sample 


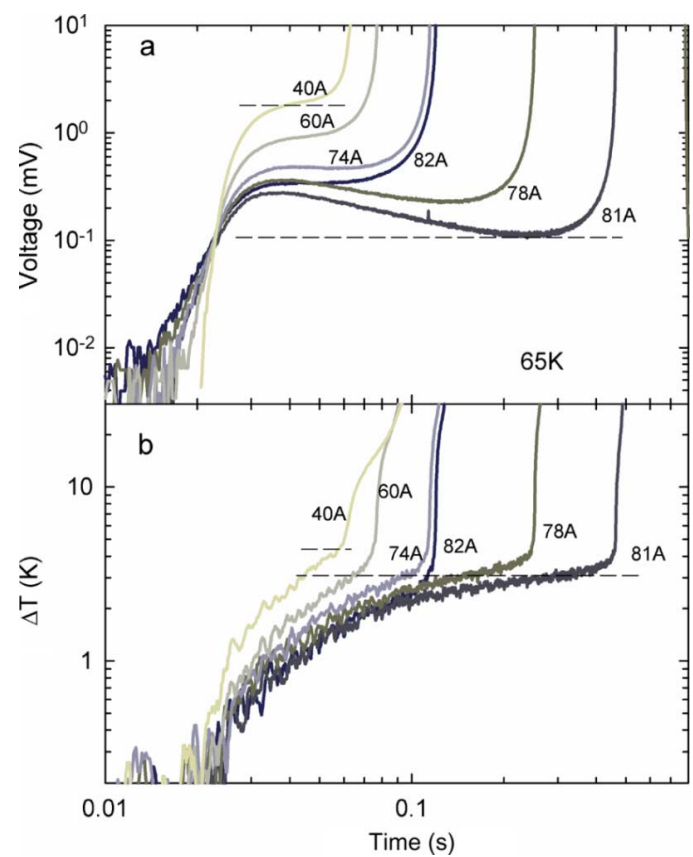

Fig. 5. (a) Hot spot voltage $V_{1}$ and (b) temperature $T_{1}$ as a function of time when a MQE pulse corresponding to the transport current between $40 \mathrm{~A}$ and $82 \mathrm{~A}$ was discharged on the conductor at $65 \mathrm{~K}$.

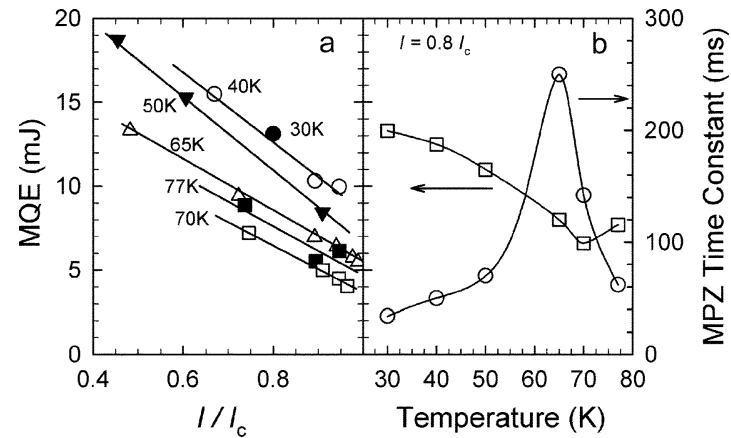

Fig. 6. (a) MQE at different temperatures as a function of reduced current $I / I_{\mathrm{C}}$, and (b) interpolated $\mathrm{MQE}$ at $80 \%$ of $I_{\mathrm{c}}$ as a function temperature. The time constant of the quasi-MPZ at high current is also shown in Fig. 6(b).

set-up has successfully resolved thermal profiles at high currents, yet more closely spaced thermocouples are required in future if thermal profiles at low currents are to be resolved.

\section{Minimum Quench Energy}

While the details of the MPZ formation and dynamic are highly relevant to the understanding of HTS quenching in a wide temperature range for current sharing, the minimum quench energy is the fundamental parameter for HTS magnet design. The MQE for the stabilizer-free 2G HTS conductor were measured at different temperatures between $30 \mathrm{~K}$ and $77 \mathrm{~K}$ with current up to 212 A. Fig. 6(a) shows that for all the temperatures, MQE decreases with increasing the transport current towards the critical current $I_{\mathrm{c}}$, and that the gradients for the relative change in MQE are quite similar. The absolute value of MQE, however, does not follow a monotonous function with temperature. From $77 \mathrm{~K}$ to $70 \mathrm{~K}$, the MQE reduces with temperature as expect for decreasing the thermal conductivity and heat capacity. Given the small cross section $\left(0.01 \mathrm{~mm}^{2}\right)$ of the sample, an MQE of
$5 \mathrm{~mJ}$ at $77 \mathrm{~K}$ is equivalent to a $0.5 \mathrm{~J} / \mathrm{mm}^{2}$, similar to that found of stabilized 2G YBCO conductors [12].

It is totally unexpected, however, that the MQE increases with decreasing temperature from $70 \mathrm{~K}$ [see Fig. 6(b) for interpolated $\mathrm{MQE}$ at $\left.0.8 I_{\mathrm{C}}\right]$. While the underlying mechanisms for this unusual behavior are yet to be explained, it is worth noting the following. Firstly, there is a marked change in the MPZ dynamics around $70 \mathrm{~K}$ when the time constant of the quasi-MPZ becomes much longer, displaying a peak mirroring the minimum in MQE. Secondly, the thermal conductivity (Fig. 2) also exhibits a similar minimum at about $65 \mathrm{~K}$ before increasing to a peak value at $25 \mathrm{~K}$. In spite of a fast reducing heat capacity, as the result of the interplay between enhanced heat diffusion and the further extended temperature range for current sharing, it is perhaps possible for the superconductor to become more stable at lower temperatures and have a higher MQE.

\section{CONCLUSION}

As postulated in previous studies [7], [8], the wide temperature range of current sharing for HTS at low temperatures does result in quench characteristics drastically different from those of the LTS. This paper has highlighted two issues to which the existing theory cannot be applied directly. Firstly, the uncertainty and non-linearity in the quench voltage of the minimum propagation zone makes it difficult to determine the size of MPZ. Secondly, the minimum quench energy MQE was found to increase with decreasing temperatures to below $70 \mathrm{~K}$, contrary to the expectation of accepted wisdom. Obviously further theoretical and experimental works are necessary to advance our understanding of the quench behavior of HTS.

\section{REFERENCES}

[1] M. N. Wilson, Superconducting Magnets. Oxford: Clarendon, 1983.

[2] Y. Iwasa, Case Studies in Superconducting Magnets. New York: Kluwer Academic, 1994.

[3] L. Dresner, Stability of Superconductors. New York: Kluwer Academic, 2002.

[4] R. B. Banks, Growth and Diffusion Phenomena: Mathematical Frameworks and Applications. Berlin: Springer, 1994.

[5] T. Huang, A. Johnston, Y. Yang, C. Beduz, and C. Friend, "Finite element modeling of thermal stability and quench propagation in a pancake coil of PbBi2223 tapes," IEEE Trans. App. Supercond., vol. 15, pp. 1647-1650, June 2005.

[6] E. Martínez, F. Lera, M. Martinez-López, Y. Yang, S. I. Schlachter, P. Lezza, and P. Kováč, "Quench development and propagation in metal/ $\mathrm{MgB}_{2}$ conductors," Supercond. Sci. Technol., vol. 19, pp. 143-150, Jan. 2006.

[7] E. Martínez, E. A. Young, M. Bianchetti, O. Muñoz, S. I. Schlachter, and Y. Yang, "Quench onset and propagation in $\mathrm{Cu}$-stabilized multifilament $\mathrm{MgB}_{2}$ conductors," Supercond. Sci. Technol., vol. 21, p. 025009 , 2008, (8pp).

[8] T. Huang, E. Martínez, C. Friend, and Y. Yang, "Quench characteristics of HTS conductors at low temperatures," IEEE Trans. Appl. Supercond., vol. 18, pp. 1317-1320, 2008.

[9] J. Lu, E. S. Choi, and H. D. Zhou, "Physical properties of Hastelloy C-276TM at cryogenic temperatures," J. Appl. Phys., vol. 103, p. 064908, Mar. 2008, (6pp).

[10] R. Grabovickic, J. W. Lue, M. J. Gouge, J. A. Demko, and R. C. Duckworth, "Measurements of temperature dependence of the stability and quench propagation of a 20-cm-long RABiTS Y-Ba-Cu-O tape," IEEE Trans. App. Supercond., vol. 13, pp. 1726-1730, June 2003.

[11] F. Trillaud, H. Palanki, U. P. Trociewitz, S. H. Thompson, H. W. Weijers, and J. Schwartz, "Normal zone propagation experiments on HTS composite conductors," Cryogenics, vol. 43, pp. 271-279, 2003.

[12] X. Wang, U. P. Trociewitz, and J. Schwartz, "Near-adiabatic quench experiments on short $\mathrm{YBa}_{2} \mathrm{Cu}_{3} \mathrm{O}_{7-\mathrm{d}}$,"J. Appl. Phys., vol. 101, p. 053904, 2007, 101, (10pp). 